\title{
Effect of Ovariectomy on the Proliferative Capacity of Intrahepatic Rat Cholangiocytes
}

\author{
DOMENICO ALVARO,* GIANFRANCO ALPINI, $, \S, \| ~ P A O L O ~ O N O R I, "$ ANTONIO FRANCHITTO, \\ SHANNON GLASER, ${ }^{\ddagger}$ GENE LE SAGE, ${ }^{\ddagger}$ ALESSANDRO GIGLIOZZI,* ANTONELLA VETUSCHI,ף \\ SERGIO MORINI,\# ADOLFO F. ATTILI,* and EUGENIO GAUDIO** \\ *Division of Gastroenterology, University “La Sapienza," Rome, Italy; ${ }^{*}$ Department of Internal Medicine, Medical Physiology, Scott \& White \\ Hospital, and §The Texas A\&M University System HSC, COM, and "Central Texas Veterans Health Care System, Temple, Texas; "Department \\ of Experimental Medicine (Human Clinical Anatomy), State University of L'Aquila, L'Aquila, Italy; \#University Campus Bio-Medico of Rome; and \\ **Departments of Anatomy and Clinical Medicine, University "La Sapienza," Rome, Italy
}

Background \& Aims: We evaluated the effects of ovariectomy (OVX) and estrogen replacement treatment on cholangiocyte proliferation induced by bile duct ligation (BDL). Methods: BDL (2 weeks) was performed in ovariectomized rats and the proliferative and apoptotic activity compared with normal, with BDL control rats, and with $\mathrm{BDL} \pm$ OVX rats treated with 17- $\beta$ estradiol. Results: OVX induced a significant $(P<0.01)$ reduction of bile duct mass in BDL rats. The reduction of bile duct mass induced by OVX was associated with a decreased expression of estrogen receptor (ER)- $\alpha$ (2.5-fold) and, mainly, ER- $\beta$ (35-fold). Proliferating cellular nuclear antigen (PCNA) expression in cholangiocytes was impaired by OVX, indicating depression of proliferation, whereas terminal deoxynucleotidyl transferase-mediated deoxyuridine triphosphate nick-end labeling (TUNEL) and Fas positivity were markedly enhanced, indicating activation of Fas-mediated apoptosis. Administration of 17- $\beta$ estradiol during BDL in OVX rats induced a normalization of bile duct mass, ER expression, cholangiocyte proliferation, and apoptosis (Fas and TUNEL) in comparison with untreated BDL rats. Conclusions: Our findings support the role of endogenous estrogens in sustaining the enhanced proliferative and secretory activities of cholangiocytes in cholestasis. On the basis of these data, the hypothesis of an estrogenic functional deficiency in chronic cholestatic liver diseases should merit careful attention.

$\mathrm{C}$ holangiocytes, the epithelial cells lining the intrahepatic biliary tree, are the target of damage in a group of chronic cholestatic liver diseases (cholangiopathies) recently classified as vanishing bile duct syndromes (VBDS). ${ }^{1-6}$ These diseases are characterized by the progressive disappearance of intrahepatic bile ducts, which leads to a severe ductopenic condition in terminal stages. ${ }^{1-6}$ Cholangiocyte proliferation (another characteristic feature of VBDS), influences, as a repair and compensatory mechanism, the outcome of the disease and its evolution toward the terminal ductopenic stage. ${ }^{1-6}$ For this reason, a number of recent studies have focused on agents and mechanisms involved in the modulation of cholangiocyte proliferation. ${ }^{6-9}$

Estrogens are important inducers of cell growth and differentiation ${ }^{10-12}$; however, until a few years ago, no information was available on the role of estrogens and their receptors on the modulation of cholangiocyte proliferation. This is a very important topic, because most VBDS, especially primary biliary cirrhosis, predominate in women. ${ }^{1-6}$ In addition, indirect evidence suggests the occurrence of estrogenic dysfunction in primary biliary cirrhosis, including increased incidence of menstrual irregularities, hysterectomy, breast cancer, and postmenopausal osteoporosis. ${ }^{13-17}$ The latter (a sign of estrogen deficiency) benefits from estrogen replacement treatment. ${ }^{18,19}$ Furthermore, marked alterations of estrogen hepatic metabolism occur in cholestasis, which is one of the hallmarks of cholangiopathies, caused by the decreased hepatic levels of $\mathrm{P} 450$-dependent microsomal enzymes, with a consequently enhanced estradiol serum level. ${ }^{20}$ We have recently shown the following: (1) Rat cholangiocytes express both estrogen receptor (ER)- $\alpha$ and ER- $\beta$ subtypes, which are overexpressed during cholangiocyte proliferation induced by BDL; (2) estrogen receptor antagonists impair BDL-induced cholangiocyte proliferation; and (3) $17-\beta$ estradiol stimulates in vitro cholangiocyte proliferation. Together, these findings indicate that estrogens and their receptors modulate

Abbreviations used in this paper: BDL, bile duct ligation; CK-19, cytokeratin-19; ER, estrogen receptors; IHC, immunohistochemical; OVX, ovariectomy; PCNA, proliferating cellular nuclear antigen; TUNEL, terminal deoxynucleotidyl transferase-mediated deoxyuridine triphosphate nick-end labeling; VBDS, vanishing bile duct syndrome.

(C) $\mathbf{2 0 0 2}$ by the American Gastroenterological Association 0016-5085/02/\$35.00 doi:10.1053/gast.2002.34169 
cholangiocyte proliferation. To get more insights into the role of estrogens in the modulation of cholangiocyte proliferation, we evaluated the effect of ovariectomy (OVX) and estrogen replacement treatment on cholangiocyte proliferation induced by BDL.

\section{Materials and Methods}

Normal female and ovariectomized Wistar rats (200$250 \mathrm{~g}$ ) were purchased from Charles River (Calco, Lecco, Italy). Reagents were purchased from Sigma Chemical Co. (St. Louis, MO) unless otherwise indicated. ICI 182,780 was purchased from Tocris (Cookson Inc., Ballwin MO). Bile duct ligation or bile duct incannulation (BDI, for bile collection) was performed as described. ${ }^{7,21}$ OVX rats were submitted to BDL 3-5 weeks after OVX. Seventeen- $\beta$ estradiol was prepared as a stock solution in dimethyl sulfoxide and administered subcutaneously, after dilution with saline (1:1000; total volume 0.5 $\mathrm{mL}$ ), at the dose of $28 \mu \mathrm{g} /$ day starting 4 days before BDL and continuing for 2 weeks thereafter. ICI 182,780 (1 mg/kg body weight per day) was dissolved in benzylbenzoate/castor oil mixture $(20 \% / 80 \% \mathrm{wt} / \mathrm{wt})$ and administered subcutaneously (100 $\mu \mathrm{L}$ volume) starting 2 days before BDL and continuing during 2-week BDL. The animals were killed, and liver histology, immunohistochemistry, molecular, and functional studies were performed. In preliminary experiments, shamOVX, sham-BDL, and the administration of the carrier for $17-\beta$ estradiol or ICI 182,780 did not influence any of the parameters measured. For this reason and to decrease the number of experimental groups, these studies were included in the same group (i.e., normal rats that include 3 normal rats, 3 sham-OVX rats, and 3 sham-BDL rats). Estradiol serum levels were measured by a radioimmunoassay kit (Diagnostic Products Corporation, Los Angeles, CA). The study protocols were in compliance with our institution's guidelines.

\section{Light Microscopy and Immunohistochemical Studies on Liver Samples}

For light microscopy, small liver fragments $(0.5 \mathrm{~cm})$ were fixed in buffered formalin for $2-4$ hours, embedded in low-temperature fusion paraffin $\left(55^{\circ} \mathrm{C}-57^{\circ} \mathrm{C}\right)$ and $3-4 \mu \mathrm{m}$ sections stained with hematoxylin-eosin and Masson's trichrome stains. For immunohistochemical (IHC), sections were mounted on glass slides coated with $0.1 \%$ poly-L-lysine. After deparaffination, endogenous peroxidase activity was blocked by a 30-minute incubation in methanolic hydrogen peroxide $(2.5 \%)$. The endogen biotin was then blocked by biotin Blocking System (code X0590; Dako, Copenhagen, Denmark), according to the instructions supplied by the vendor. Sections were hydrated in graded alcohol and rinsed in phosphate-buffered saline (PBS; $\mathrm{pH}$ 7.4) before applying the primary antibody. Sections were incubated overnight at $4^{\circ} \mathrm{C}$ with monoclonal antibodies for the following: cytokeratin 19 (CK-19; Dako), ${ }^{22}$ PCNA (PC10; Dako), Fas (M-20)G (Santa Cruz Inc., Santa Cruz, CA), and with polyclonal antibodies for ER- $\beta$ (PA1.1-310, Affinity Bioreagents, Inc., Golden, CO). Samples were then rinsed with PBS for 5 minutes, incubated for 10 minutes at room temperature with secondary biotinylated antibody (Dako LSAB2, code K0675; Dako), with Dako ABC (code K0675; Dako), and, finally, developed with 3-3' diaminobenzidine (DAB). For ER- $\alpha$ detection, we used a Dako Catalyzed Signal Amplification (code K1500; Dako) according to the instructions supplied by the vendor. Samples were incubated overnight at $4{ }^{\circ} \mathrm{C}$ with the monoclonal primary antibodies: for ER- $\alpha$ subtype, a cocktail of 2 monoclonal antibodies C-314 and C-311 (50\% of each ${ }^{8}$; Santa Cruz Inc.). Sections were developed with DAB. For all immunoreactions, negative and positive controls were also included. Light microscopy and IHC microphotographs were taken by Olympus BX-5 1 Light Microscopy with a Videocam (Spot Insight, Diagnostic Instrument, Inc., Sterling Heights, MI) and processed with an Image Analysis System (IAS-Delta Sistemi, Roma, Italy) software. Intrahepatic ductal mass was evaluated $^{7-9}$ in 6 blocks for each liver, randomly taken from median lobe. Point count analysis was performed by determining the number of ducts stained ${ }^{7-9}$ for either $\gamma$-glutamyltranspeptidase $(\boldsymbol{\gamma}-\mathrm{GT})$ or CK-19, present in each coded section (2-3- $\mu \mathrm{m}$ thickness), obtained randomly. Light microscopy images $(10 \times$ or $20 \times)$ were analyzed by using Image Analysis System software (IAS-Delta Sistemi). For in situ detection of apoptosis on the single cell, we used the method of end labeling mediated by deoxynucleotidyl transferase (TdT) and labeling (terminal deoxynucleotidyl transferase-mediated deoxyuridine triphosphate nick-end labeling; TUNEL) method (Apotag Kit; Oncor, Gaithersburg, MD). Negative and positive controls were included in each bath of slide. Morphological measurements were performed in a blind fashion by 3 independent pathologists (E.G., P.O., A.F.) coming from 2 different institutions, with an agreement always higher than $90 \%$.

\section{Western Blot Analysis of Proliferating Cellular Nuclear Antigen in Purified Cholangiocytes}

Pure preparations of cholangiocytes were obtained as described $^{7-9}$ from normal, BDL, BDL + OVX, and BDL + OVX $+17-\beta$ estradiol-treated rats. Cholangiocyte proliferation was assessed by measurement of proliferating cellular nuclear antigen (PCNA) protein expression by immunoblots as described. ${ }^{7-9}$ The intensity of the bands was determined by scanning video densitometry using the ChemiImager 4000 low-light imaging system (Alpha Innotech Corp., San Leandro, CA). Purity of cholangiocyte preparation was assessed by $\gamma$-glutamyltransferase (GGT) staining, ${ }^{7-9}$ and contamination from hepatocytes was controlled by glucose-6-phosphatase ${ }^{23}$ staining. Virtually, all isolated cells were $\gamma$-GT positive, and no glucose-6-phosphatase positive cell was found in cell preparations from both normal and BDL livers, thus excluding hepatocyte contamination. 


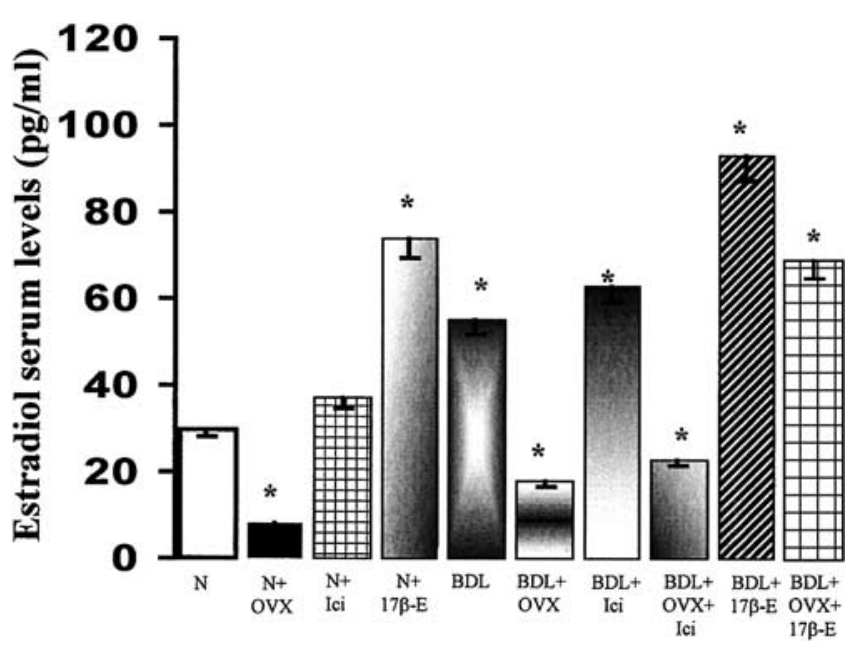

Figure 1. Measurement of estradiol serum levels. OVX decreased more than 3-fold estradiol serum levels in normal and $\mathrm{BDL}$ rats ( $\mathrm{N}$ vs. $\mathrm{N}+\mathrm{OVX}$ and $\mathrm{BDL}$ vs. BDL + OVX). 17- $\beta \mathrm{E}$ administration induced a marked increase of estradiol serum levels ( $N$ vs. $N+17-\beta E$, BDL vs. $\mathrm{BDL}+17-\beta \mathrm{E}, \mathrm{BDL}+\mathrm{OVX}$ vs. $\mathrm{BDL}+\mathrm{OVX}+17-\beta \mathrm{E}) . * P<0.05$ vs. the other groups. Data are mean \pm SE of $n=5-9$. Abbreviations: $N$, normals; OVX, ovariectomy; BDL, bile duct ligation; ICI, ICI 182,780; $17-\beta \mathrm{E}, 17-\beta$ estradiol.

\section{Statistical Analysis}

All data are expressed as mean $\pm \mathrm{SE}$. The differences between groups were evaluated by analysis of variance (ANOVA).

\section{Results}

Effect of OVX, BDL, and Estrogen Replacement Treatment on Estradiol Serum Levels

OVX induced a 3 -fold decrease in the estradiol serum levels of normal and BDL rats $(P<0.01$, Figure 1). However, 17- $\beta$ estradiol administration induced a marked $(P<0.01$, Figure 1$)$ increase in estradiol serum levels of normal and BDL rats. When 17- $\beta$ estradiol was administered during BDL in OVX rats, serum estradiol levels rose higher $(P<0.05$, Figure 1$)$ than BDL controls. These findings indicate that the administered amount of $17-\beta$ estradiol was effective in restoring the estrogen serum levels decreased by OVX.

\section{Effects of OVX and Estrogen Replacement Treatment on Bile Duct Mass, Cell Proliferation, and Apoptosis}

Neither OVX nor treatment with ICI 182,780 or $17-\beta$ estradiol induces changes of intrahepatic bile duct volume in normal rats (Figure 2). Parallel with previous studies, ${ }^{1-9}$ 2-week BDL induced a marked increase in bile duct volume in comparison with normal rats $(6.94 \%$ $\pm 0.26 \%$ vs. $0.21 \% \pm 0.09 \%$, respectively, $P<0.01$
Figure 2). However, when BDL was performed in rats with OVX for 3-5 weeks, bile duct volume was decreased by approximately $30 \%(5.05 \% \pm 0.34 \%, P<$ 0.02 ) in comparison with control BDL rats (Figure 2), indicating that induction of bile duct proliferation by BDL was impaired by the ablation of ovaric function. Administration of the selective antiestrogen ICI 182,780 during $\mathrm{BDL}$ induced a decrease in bile duct volume $(-50 \% ; P<0.01)$, which was more pronounced $(P<$ $0.05)$ than that observed with OVX. When ICI 182,780 was administered during BDL in OVX rats, bile duct volume was further decreased $(-85 \% ; P<0.01)$ in comparison with BDL + OVX or BDL + ICI 182,780 (Figure 2). 17- $\beta$ estradiol treatment induced no effect on bile duct volume of BDL rats. However, when 17- $\beta$ estradiol was administered (starting 4 days before BDL) during 2-weeks BDL in OVX rats, bile duct volume $(7.6 \% \pm 0.55 \%)$ became similar to that of control BDL rats (Figure 2). This indicates that the restoration of estrogenic function through exogenous administration of $17-\beta$ estradiol induces a normalization of the proliferative response of the intrahepatic biliary epithelium to BDL.

\section{Effect of OVX and Estrogen Replacement Treatment on the IHC Expression of ER- $\alpha$ and ER- $\beta$, Cholangiocyte Proliferation, and Apoptosis}

OVX, ICI 182,780, and 17- $\beta$ estradiol did not influence the IHC expression of ER- $\alpha$, ER- $\beta$, PCNA, Fas, or TUNEL positivity in normal rats (Figures 3-8). As far as immunohistochemistry for ER is concerned, the stain for ER- $\alpha$ was nuclear, whereas that for ER- $\beta$ was cytoplasmic given the characteristic of the antibody used that resulted in similar cytoplasmic staining in positive control tissues, including the uterus. BDL induced a marked $(P<0.01)$ increase in the percentage of cholangiocytes positive for ER- $\alpha$ or ER- $\beta$ in comparison with normal rats (Figures 3 and 4 ). However, the increase of ER- $\beta$ positivity (35-fold) was much more pronounced than that of ER- $\alpha$ (3.5-fold). When BDL was performed in OVX rats, the percentage of cholangiocytes positive for ER- $\alpha$ or ER- $\beta$ decreased $(P<0.01)$ in comparison with BDL rats $(-32 \%$ and $-45 \%$, respectively; Figures 3 and 4). The administration of the antiestrogen ICI 182,780 during BDL decreased ER- $\alpha$ positivity (percentage cholangiocytes) by a similar extent $(-31 \%$; $P<$ 0.01) than OVX, but it induced a dramatic effect on ER- $\beta$ expression, which was decreased by $94 \%(P<$ 0.01 ) and became similar to normal rats (Figures 3 and 4). When ICI 182,780 was administered during BDL in OVX rats, the IHC expression of ER- $\alpha$ or ER- $\beta$ was 


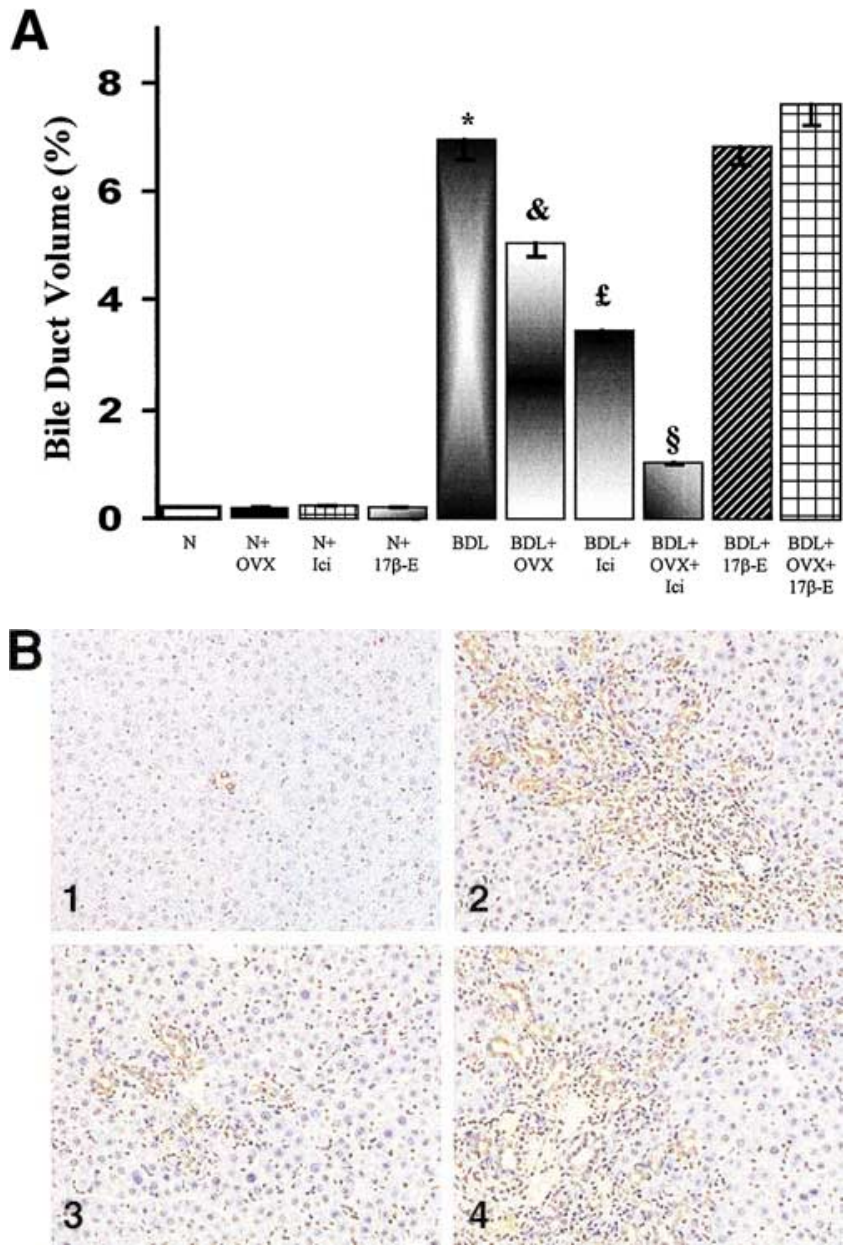

Figure 2. Bile duct volume evaluated by quantitative morphometry after immunostaining with CK-19. (A) Bar graph. OVX treatment with ICI 182,780 and $17-\beta$ estradiol induced no significant changes in bile duct volume in normal rats. Two-week BDL induced a very large increase of bile duct volume in comparison with normal rats $(* P<$ 0.01). When BDL was performed in OVX rats, bile duct volume was decreased in comparison with control BDL rats $(\& P<0.02)$. ICl 182,780 administered during BDL induced a decrease in bile duct volume, which was more pronounced than that observed with OVX (i.e., ICl + BDL vs. BDL + OVX; ${ }^{£} P<0.05$ ). When ICI 182,780 was administered during $\mathrm{BDL}$ in $\mathrm{OVX}$ rats $(\mathrm{BDL}+\mathrm{OVX}+\mathrm{ICl})$, bile duct volume was further decreased in comparison with $\mathrm{BDL}+\mathrm{OVX}$ or $\mathrm{BDL}+\mathrm{ICl} 182,780(\S P<0.01) \cdot 17-\beta$ estradiol treatment had no effect on bile duct volume of BDL rats (BDL $+17-\beta E$ vs. BDL), but, when administered during 2 weeks of BDL in OVX rats, it induced a normalization (i.e., similar to control BDL) of bile duct volume (BDL + OVX $+17-\beta$ E vs. BDL + OVX). Data are mean \pm SE of $n=5-9$. For abbreviations see Figure 1 legend. (B) Pictures of (1) normal, (2) BDL, (3) $\mathrm{BDL}+\mathrm{OVX}+\mathrm{ICl}$, and (4) BDL + OVX + 17- $\beta$ estradiol are shown (original magnification $20 \times$ ).

further decreased ( $-52 \%$ and $-97 \%$, respectively) in comparison with BDL + OVX or BDL + ICI 182,780treated rats (Figures 3 and 4 ). 17- $\beta$ estradiol administration showed no effect in BDL rats but completely restored (similar to BDL) the IHC expression of ER- $\alpha$ and ER- $\beta$ when administered during BDL in OVX rats (Figures 3 and 4).
The decreased proliferative response of intrahepatic bile ducts to BDL in OVX rats was caused by both impaired cholangiocyte proliferation and enhanced Fasmediated apoptotic cholangiocyte death. The impaired cholangiocyte proliferation in OVX rats was showed by immunohistochemistry for PCNA (Figure 5). The percentage of PCNA-positive cholangiocytes, in fact, was significantly lower in BDL + OVX than in BDL rats
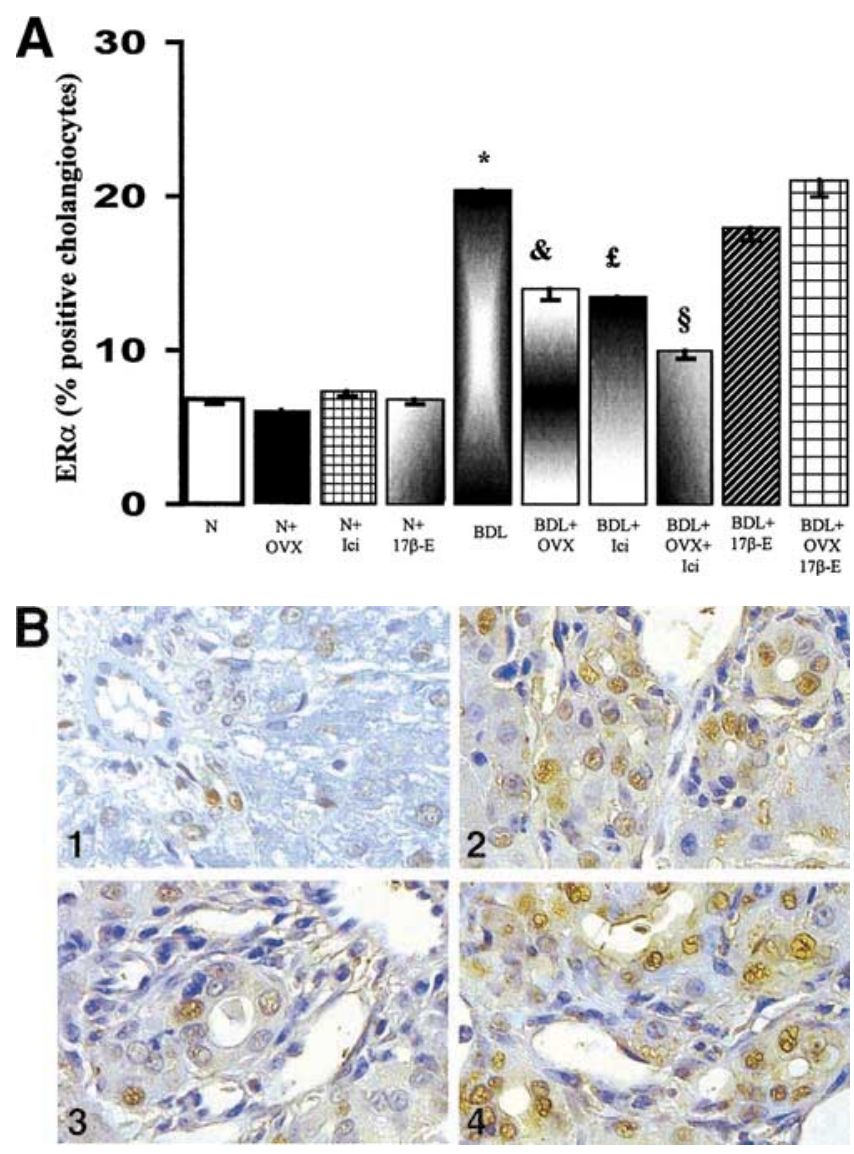

Figure 3. Immunohistochemistry for estrogen receptor ER- $\alpha$ in the liver. (A) Bar graph. The percentage of cholangiocyte positive for ER- $\alpha$ was similar between normal, normal + OVX rats, normal $+\mathrm{ICl}$ 182,780 , and normal $+17-\beta$ estradiol. BDL induced a marked increase in the percentage of cholangiocytes positive for ER- $\alpha$ in comparison with normal rats (BDL vs. $\mathrm{N}$; $* P<0.01$ ). When $\mathrm{BDL}$ was performed in OVX rats, the percentage of cholangiocytes positive for ER- $\alpha$ decreased in comparison with BDL rats (BDL + OVX vs. BDL; ${ }^{\&} P<0.01$ ). The administration of the antiestrogen ICI 182,780 during $\mathrm{BDL}$ decreases ER- $\alpha$ positivity (percentages cholangiocytes) to a similar extent than OVX (BDL + ICl vs. BDL + OVX and vs. BDL; ${ }^{£} P<$ 0.01). When $\mathrm{ICI} 182,780$ was administered during $\mathrm{BDL}$ in OVX rats, the IHC expression of ER- $\alpha$ was further decreased $(B D L+I C I+O V X$ vs. $\mathrm{BDL}+\mathrm{ICl}$ and vs. BDL + OVX; $\$ P<0.01) .17-\beta$ estradiol administration showed no effect in BDL rats but completely restored (i.e., similar to BDL) the IHC expression of ER- $\alpha$ when administered during $\mathrm{BDL}$ in OVX rats (BDL + OVX $+17-\beta \mathrm{E}$ vs. $\mathrm{BDL}+\mathrm{OVX}$ and vs. $\mathrm{BDL})$. Data are mean \pm SE of $n=5-9$. For abbreviations see Figure 1 legend. (B) Pictures of (1) normal, (2) BDL, (3) BDL + OVX + ICI, and (4) $\mathrm{BDL}+\mathrm{OVX}+17-\beta$ estradiol are shown (original magnification $40 \times$ ). 

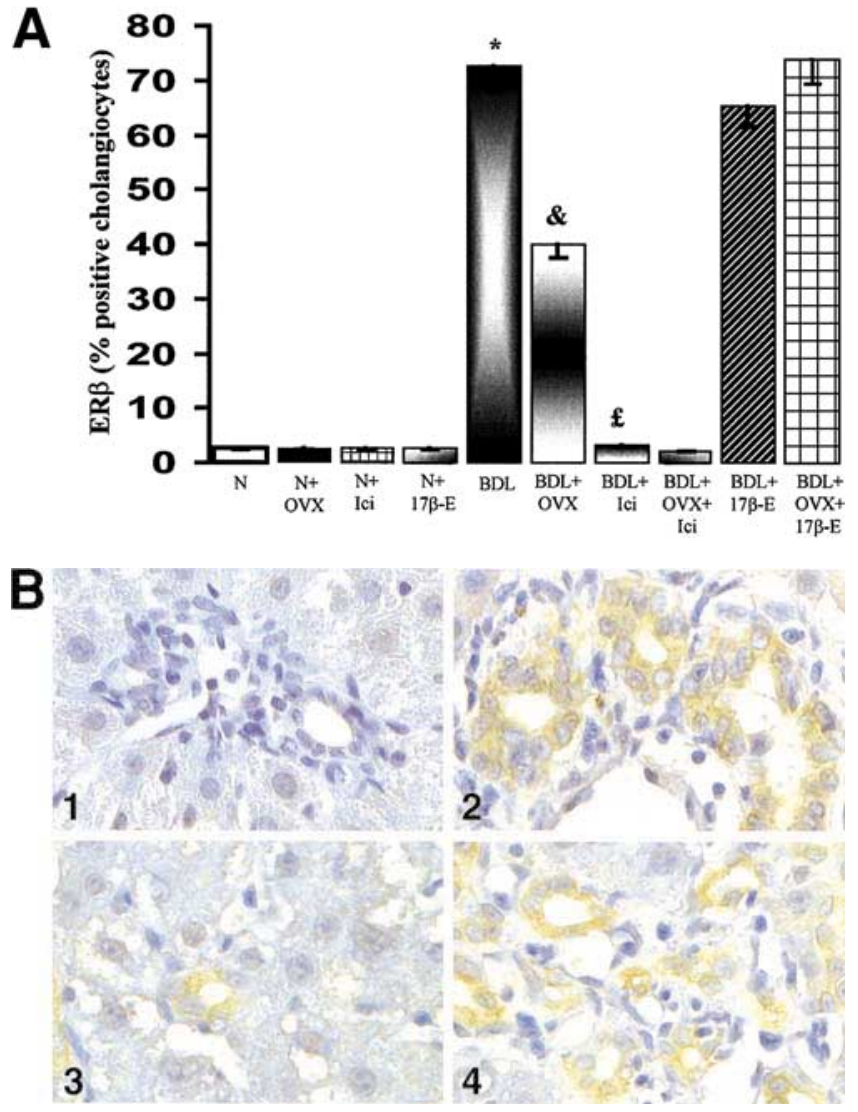

Figure 4. Immunohistochemistry for estrogen receptor ER- $\beta$ in liver sections. (A) Bar graph. The percentage of cholangiocyte positive for ER- $\beta$ was similar between normal, normal + OVX rats, normal + ICl 182,780 , and normal $+17-\beta$ estradiol. BDL induced a marked increase in the percentage of cholangiocytes positive for ER- $\beta$ in comparison with normal rats (BDL vs. $\mathrm{N} ;{ }^{*} P<0.01$ ). When BDL was performed in OVX rats, the percentage of cholangiocytes positive for ER- $\beta$ decreased in comparison with BDL rats (BDL + OVX vs. BDL; ${ }^{\&} P<0.01$ ). The administration of the antiestrogen ICI 182,780 during BDL induced a dramatic decrease of ER- $\beta$ positivity in cholangiocytes ( ${ }^{\ddagger} P<0.01$ vs. BDL or BDL $+\mathrm{OVX}$ ). $17-\beta$ estradiol administration showed no effect in BDL rats but completely restored the immunohistochemical expression of ER- $\beta$ (i.e., similar to BDL) when administered during BDL in OVX rats (BDL + OVX $+17-\beta \mathrm{E}$ vs. BDL + $O V X)$. Data are mean $\pm S E$ of $n=5-9$. For abbreviations see Figure 1 legend. (B) Pictures of (1) normal, (2) BDL, (3) BDL + OVX + ICl, and (4) $\mathrm{BDL}+\mathrm{OVX}+17-\beta$ estradiol are shown (original magnification $40 \times)$.

$(P<0.05 ;$ Figure 5). The antiestrogen ICI 182,780 induced a decrease $(P<0.05)$ of PCNA positivity in cholangiocytes, which was similar to the effect observed in OVX rats and displayed an additive effect with OVX when administered during BDL (Figure 5). 17- $\beta$ estradiol treatment showed no effect on BDL rats but completely normalized PCNA positivity in BDL + OVX rats where PCNA positivity became similar to untreated BDL rats (Figure 5). In selected experiments, PCNA protein expression was also measured by immunoblots in pure cholangiocytes from normal $(\mathrm{n}=3)$,
$\mathrm{BDL}(\mathrm{n}=3), \mathrm{BDL}+\mathrm{OVX}(\mathrm{n}=3)$, or $\mathrm{BDL}+\mathrm{OVX}$ $+17-\beta$ estradiol $(\mathrm{n}=3)$ treated rats (Figure 6$)$. Consistent with IHC data, BDL induced a marked $(P<$ $0.01)$ increase in PCNA protein expression in cholangiocytes, which was decreased $(P<0.01$; Figure 6$)$ when BDL was performed in OVX rats. The effect of OVX on PCNA protein expression in BDL rats was completely reverted by $17-\beta$ estradiol administration $(P<0.01$; $\mathrm{BDL}+\mathrm{OVX}+17-\beta$ estradiol vs. BDL + OVX; Figure 6).

Positivity for Fas and TUNEL involved less than 2.5\% cholangiocytes of BDL rats (Figures 7 and 8 ). When BDL
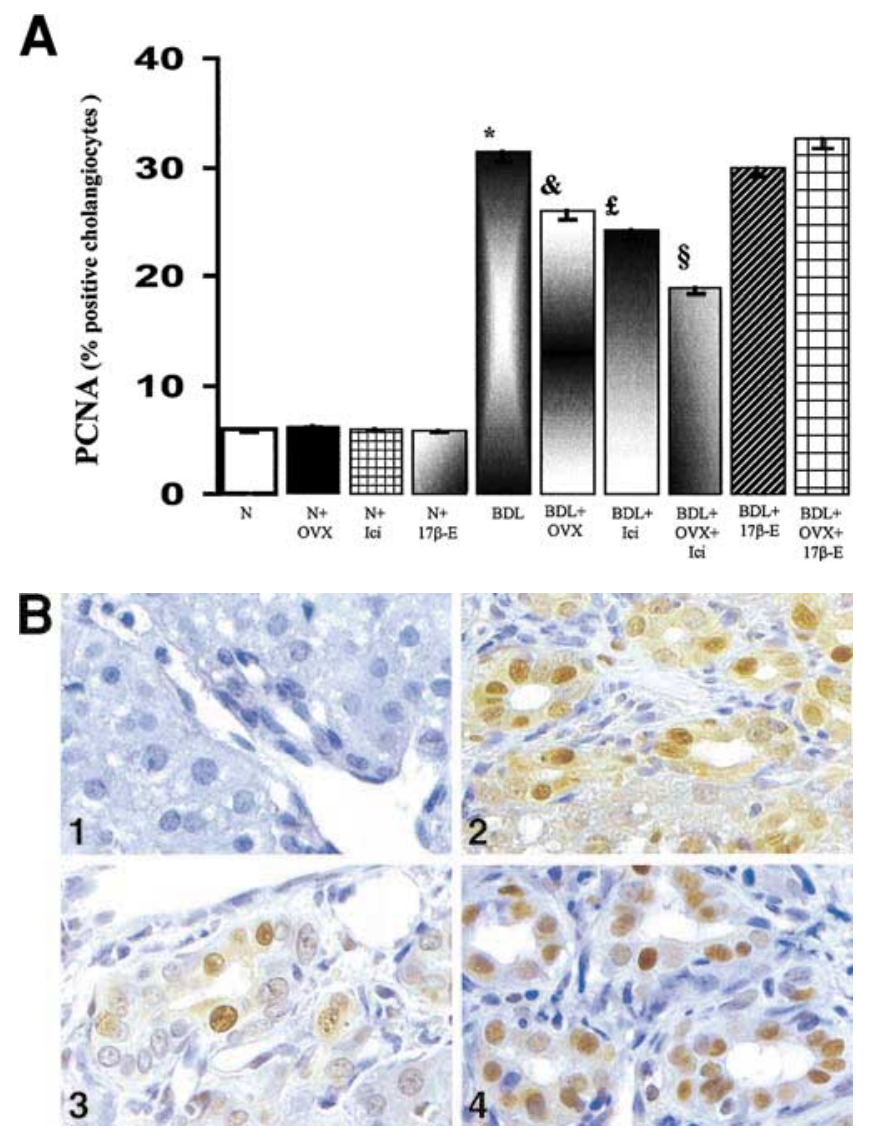

Figure 5. Immunohistochemistry for PCNA in liver sections. (A) Bar graph. The percentage of cholangiocyte positive for PCNA was similar between normal, normal + OVX rats, normal + ICI 182,780, and normal $+17-\beta$ estradiol. BDL induced a marked increase of the percentage PCNA positive cholangiocytes (BDL vs. N; $* P<0.01$ ), which was decreased when BDL was performed in OVX rats (BDL + OVX vs. BDL; \& $P<0.05)$. The antiestrogen ICl 182,780 decreases PCNA positivity in cholangiocytes of BDL rat livers (BDL + ICl vs. BDL; $\left.{ }{ }_{P}<0.05\right)$ to a similar extent than the effect observed by OVX and displayed an additive effect with OVX (BDL + OVX + ICI vs. BDL + ICl or BDL + OVX; $\$ P<0.05)$. 17- $\beta$ estradiol treatment showed no effect on $\mathrm{BDL}$ rats but completely normalized (i.e., similar to $\mathrm{BDL}$ controls) PCNA positivity in BDL + OVX (BDL + OVX 17- $\beta$ E vs. BDL and vs. $\mathrm{BDL}+\mathrm{OVX})$. Data are mean $\pm \mathrm{SE}$ of $\mathrm{n}=5-9$. For abbreviations see Figure 1 legend. (B) Pictures of (1) normal, (2) BDL, (3) BDL + OVX + $\mathrm{ICl}$, and (4) BDL $+\mathrm{OVX}+17-\beta$ estradiol are shown (original magnification $40 \times)$. 


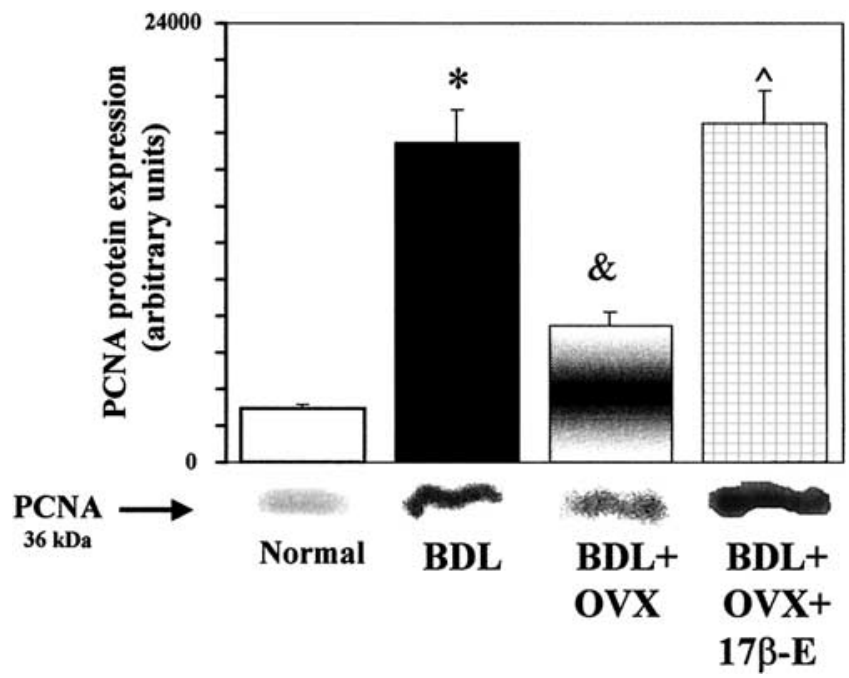

Figure 6. Western blot analysis of PCNA in isolated cholangiocytes. Cholangiocytes isolated from BDL rats showed a PCNA protein expression markedly higher than normal rats. When BDL was performed in OVX rats, PCNA in cholangiocytes markedly decreased, but this effect of OVX was completely restored by 17- $\beta$ estradiol replacement treatment. Mean $\pm \mathrm{SE}$ of $\mathrm{n}=3$ are shown in the bar graph. $* P<0.01$ vs. normal; $\& P<0.01$ vs. $\mathrm{BDL} ; P<0.01$ vs. $\mathrm{BDL}+\mathrm{OVX}$.

was performed in OVX rats, there was a significant $(P<$ $0.01)$ increase in the number of cholangiocytes expressing Fas (6\%), which were also positive by TUNEL analysis (Figures 7 and 8). Treatment of BDL rats with the antiestrogen ICI 182,780 resulted in a dramatic increase $(P<0.01)$ of Fas and TUNEL positivity (more than $60 \%$ cholangiocytes, which was only slightly further increased when the antiestrogen was administered in OVX rats during BDL; Figures 7 and 8). The administration of $17-\beta$ estradiol in BDL rats induced no effect on Fas and TUNEL positivity, but, when the estrogen replacement treatment was performed in OVX rats during BDL, a complete normalization of Fas and TUNEL was observed, which was similar to BDL rats in the absence of any treatment (Figures 7 and 8). These findings suggest that OVX induced a slight activation of Fas-mediated apoptosis in BDL rats, which was completely reverted by estrogen replacement treatment. A dramatic activation of Fas-mediated apoptosis was observed when ER receptors were blocked by ICI 182,780.

\section{Discussion}

The study shows that (1) OVX impairs the proliferative response of intrahepatic bile ducts to experimental cholestasis (BDL) as showed by the significant lower intrahepatic bile duct volume of BDL + OVX rats than BDL controls; (2) the decreased intrahepatic bile duct mass of OVX rats after BDL was associated with decreased cholangiocyte proliferation (as shown by the lower PCNA expression) and with enhanced Fas-mediated apoptosis of proliferating cholangiocytes as shown by immunohistochemistry for Fas and by TUNEL analysis; (3) exogenous administration of $17-\beta$ estradiol in OVX rats normalizes the proliferative response of the intrahepatic bile ducts to BDL (bile duct volume) by restoring proliferation (PCNA) and ablating Fas-mediated apoptosis (Fas and TUNEL) in cholangiocytes; and (4) the negative effects of OVX on the proliferative capacity of cholangiocytes during BDL were associated with a decreased IHC expression of ER- $\alpha$ and ER- $\beta$ in proliferating cholangiocytes; this effect also was reversed by estrogen replacement treatment. Taken together, these findings are consistent with a role of endogenous estrogens in modulating cholangiocyte proliferation during experimental cholestasis.

Cholangiocyte proliferation is a typical response of the liver to bile duct obstruction, which was reproduced experimentally in the BDL rat model. ${ }^{1-6}$ In this experimental model of selective cholangiocyte proliferation, a number of factors have been considered to play a role in the induction and maintenance of proliferative response, ${ }^{6}$ including the increased endoluminal pressure and the local or systemic release of growth factors (HGF, IGF1, EGF) and cytokines (interleukin-6). ${ }^{6}$ Based on indirect and direct evidence, we have recently proposed that estrogens and their receptors play an important role in modulating cholangiocyte proliferation. ${ }^{8}$ The indirect evidence was based on the inhibitory effect of estrogen antagonists (tamoxifen and ICI 182,780) on cholangiocyte proliferation induced by $\mathrm{BDL}$, whereas the direct evidence was based on the in vitro ability of $17-\beta$ estradiol to stimulate cholangiocyte proliferation. ${ }^{8}$ In the present study, we approached the same topic by evaluating the effects of ablation of ovaric function (by OVX) on the proliferative response of intrahepatic bile ducts to BDL. Three to 5 weeks after OVX, rats underwent BDL and, subsequently, the proliferative response of cholangiocytes was compared with BDL control rats. Estradiol serum levels were measured in the different experimental groups with the evidence that the absence of ovaric function (by OVX) resulted in a 3-fold decrease of estradiol serum levels, with the residual serum estrogens originating mainly from the adrenal gland. Parallel with other studies, ${ }^{8,20}$ BDL resulted in a marked increase of estradiol serum levels because of the impaired metabolism and biliary excretion of endogenous estrogens. Estrogen serum levels were virtually doubled after OVX rats underwent BDL for 2 weeks, but still persisted 2.5 -fold lower than control BDL. Finally, the doses of $17-\beta$ estradiol administered in OVX + BDL rats were 

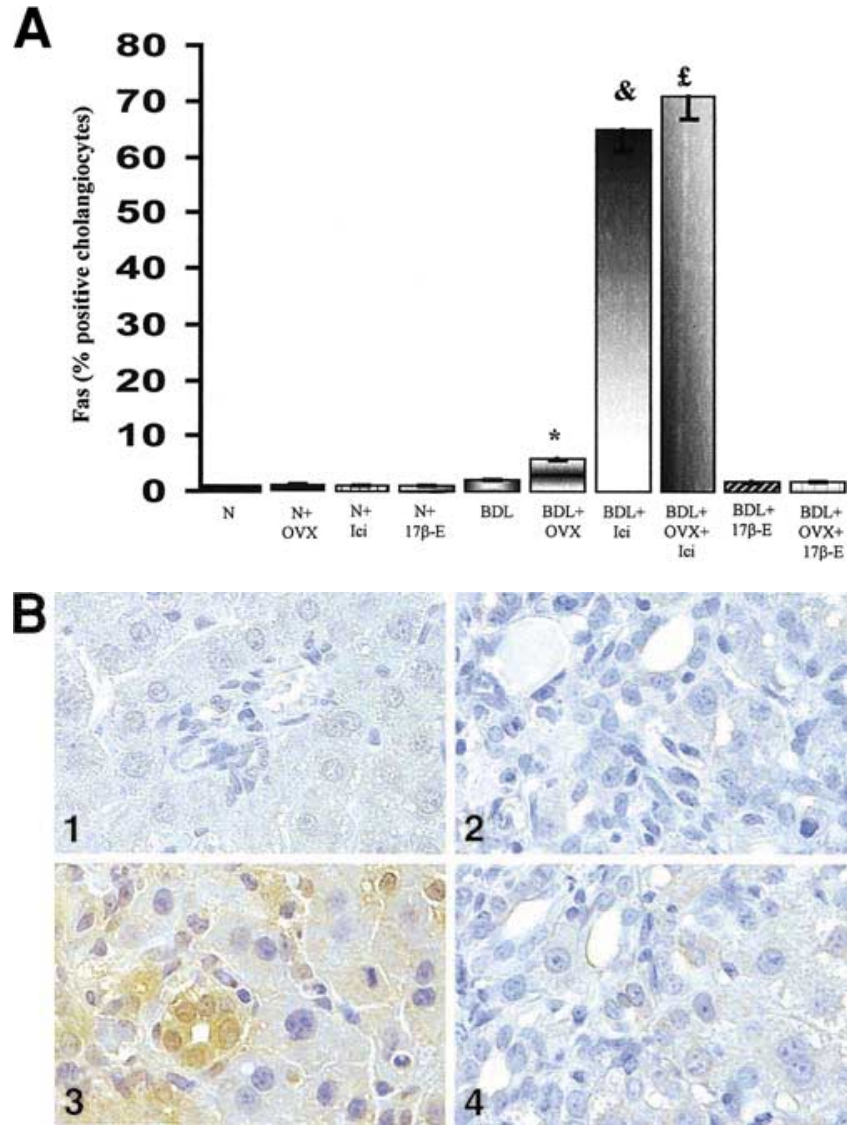

Figure 7. Immunohistochemistry for Fas in the liver. (A) Bar graph. The percentage of cholangiocytes positive for Fas was less than $2.5 \%$ cholangiocytes in normals, normals + OVX, normals + ICI, normals + $17-\beta \mathrm{E}$, and BDL rats. When BDL was performed in OVX rats, the percentage of cholangiocyte Fas positivity significantly increased (BDL + OVX vs. BDL). Treatment of BDL rats with the antiestrogen ICI 182,780 resulted in a dramatic increase of Fas positivity in cholangiocytes (BDL $+\mathrm{ICl}$ vs. BDL). The administration of $17-\beta$ estradiol in $\mathrm{BDL}$ rats induced no effect on Fas positivity (BDL $+17-\beta \mathrm{E}$ vs. $\mathrm{BDL})$, but, when the estrogen replacement treatment was performed in OVX rats during $\mathrm{BDL}$, a complete normalization of Fas positivity was observed (BDL + OVX $+17-\beta E$ vs. BDL + OVX and vs. BDL), which become similar to BDL rats in the absence of any treatment. Data are mean \pm SE of $n=5-9 .{ }^{*} P<0.05$ vs. all the other groups; ${ }^{\&} P<0.05$ vs. all the other groups but not BDL $+\mathrm{OVX}+\mathrm{ICl} ;{ }^{£} P<0.05$ vs. the other groups but not $\mathrm{BDL}+\mathrm{ICl}$. For abbreviations Figure 1 legend. $(B)$ Pictures of (1) normal, (2) BDL, (3) BDL + OVX + ICI, and (4) BDL + OVX $+17-\beta$ estradiol are shown (original magnification $40 \times$ ).

effective in increasing serum estradiol at levels similar to BDL control rats. Having shown that OVX was effective in depressing endogenous estrogen levels and that the estrogen replacement treatment was able to reverse the effects of OVX, we demonstrated that OVX induces an impaired proliferative response of the intrahepatic bile duct system to BDL. The intrahepatic bile duct volume of BDL + OVX rats was in fact $30 \%$ less than control $\mathrm{BDL}$ rats with an intact ovaric function as evaluated by quantitative morphometry. The effect of OVX was lower than that obtained by blocking endogenous estrogens with the pure ER antagonist ICI 182,780, and an almost complete block of BDL-induced expansion of bile duct volume was obtained only when the 2 treatments were combined (i.e., OVX + ICI 182,780). The impaired response of intrahepatic bile duct volume to bile duct obstruction in OVX rats was caused mainly by an impaired cell proliferation as evidenced by PCNA immunostaining and immunoblotting but also by a slight activation of Fas-mediated apoptotic cell death. This was
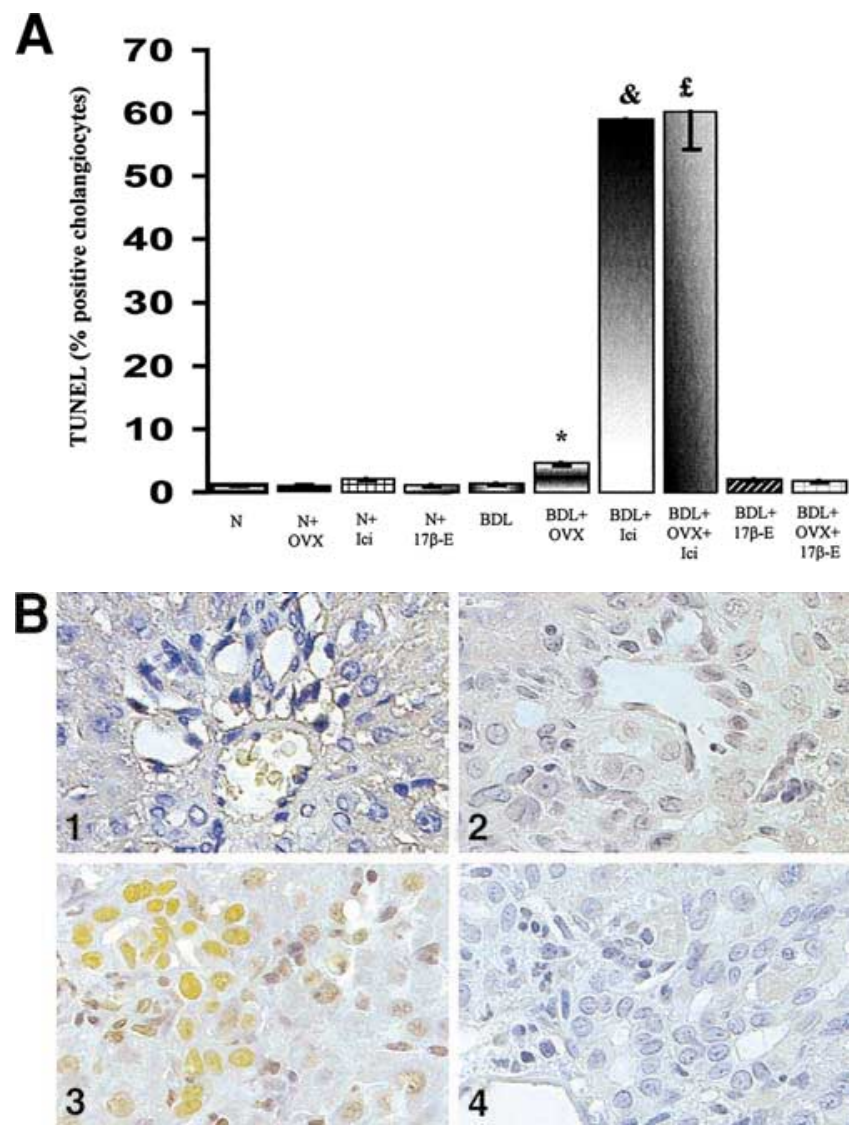

Figure 8. TUNEL analysis liver sections. (A) Bar graph. The percentage of cholangiocytes positive at the TUNEL analysis was less than $2.5 \%$ cholangiocytes in normals, normals $+\mathrm{OVX}$, normals $+\mathrm{ICl}$, normals $+17-\beta \mathrm{E}$, and $\mathrm{BDL}$ rats. When BDL was performed in OVX rats, the percentage of cholangiocyte TUNEL positivity significantly increased (BDL + OVX vs. BDL). Treatment of BDL rats with the antiestrogen $\mathrm{ICI} 182,780$ resulted in a dramatic increase of TUNEL positivity in cholangiocytes (BDL $+\mathrm{ICl}$ vs. $\mathrm{BDL}$ ), which was only slightly increased when the antiestrogen was administered during $\mathrm{BDL}$ in OVX rats $(\mathrm{BDL}+\mathrm{OVX}+\mathrm{ICl}$ vs. $\mathrm{BDL}+\mathrm{OVX})$. The administration of $17-\beta$ estradiol in BDL rats induced no effect on TUNEL positivity (BDL + 17- $\beta$ E vs. BDL), but, when the estrogen replacement treatment was performed in OVX rats during $B D L$, a complete normalization of TUNEL positivity was observed (BDL + OVX + 17- $\beta \mathrm{E}$ vs. BDL+OVX and vs. BDL), which become similar to BDL rats in the absence of any treatment. Data are mean \pm SE of $n=5-9$. $* P<0.05$ vs. all the other groups; ${ }^{\&}<0.05$ vs. all the other groups but not BDL + OVX + $\mathrm{ICl} ;{ }^{f} P<0.05$ vs. all the other groups but not $\mathrm{BDL}+\mathrm{ICl}$. For abbreviations see Figure 1 legend. (B) Pictures of (1) normal, (2) BDL, (3) $\mathrm{BDL}+\mathrm{OVX}+\mathrm{ICl}$, and (4) BDL + OVX + 17- $\beta$ estradiol are shown (original magnification $40 \times$ ). 
different from what was observed when endogenous estrogens were blocked by the pure ER antagonist ICI 182,780, where a dramatic activation of Fas-mediated apoptosis was observed. The fact that antiestrogens and specifically ICI 182,780 may induce apoptosis of target cells expressing ER has been previously shown, and this correlates with their growth inhibitory action. ${ }^{24,25}$ The proapoptotic effect of antiestrogens occurs through multiple mechanisms other than the block of the estrogen mitogenic effect, including down-regulation of the antiapoptotic protein bcl-2 or inhibition of calmodulindependent survival signals. ${ }^{24-26}$ The latter could be of relevance in proliferating cholangiocytes, where intracellular $\mathrm{Ca}^{2+}$, protein kinase $\mathrm{C}$, and calmodulin-dependent signals play a role in sustaining cholangiocyte proliferative machinery. 7,9 Thus, in evaluating the role of endogenous estrogens as modulators of the cholangiocyte proliferative response to experimental cholestasis, the effects of OVX seem to be different from those of antiestrogens, because the latter are characterized by a marked proapoptotic effect.

The effect of OVX on cholangiocyte proliferation was associated with a $40 \%-50 \%$ lower IHC expression of both ER- $\alpha$ and ER- $\beta$, indicating that the down-regulation of both receptor subtypes as a consequence of OVXinduced estrogen depletion is the mechanism involved. In contrast, the antiproliferative effect of ICI 182,780 is associated with an almost complete ablation of ER- $\beta$, and this is consistent with recent data suggesting a central role played by ER- $\beta$ in mediating growth-inhibitory action of antiestrogens. ${ }^{27}$ The pronounced effect of the antiestrogen ICI 182,780 on both ER- $\beta$ expression and apoptosis could suggest a role of this receptor (once activated by the selective agonists) in the down-regulation of apoptotic machinery. However, in some cell types, ER- $\beta$ plays an antiproliferative action, ${ }^{28}$ antagonizing the positive modulatory effects of ER- $\alpha$. In cholangiocytes, the present and previous studies ${ }^{8}$ suggest that both ER- $\alpha$ and ER- $\beta$ play a role in mediating the positive modulatory effects of endogenous estrogens on cholangiocyte proliferation. Finally, we showed that exogenous administration of $17-\beta$ estradiol completely reversed the effects of OVX on bile duct mass, ER expression, cell proliferation, and apoptosis. These findings support the specificity of OVX effects, which are due to depressed estrogen levels and not to other confounding factors. Regarding this, it is well known that estrogens may induce cholestasis by affecting the function and protein expression of different transporters in hepatocytes, ${ }^{29}$ thus, it may be argued that the effect of $17-\beta$ estradiol could be a consequence of its cholestatic effect.
We may exclude the latter possibility, because, in our study, estrogen replacement treatment was performed using a dose 30-50-fold lower than that used to induce experimental cholestasis. In addition, estradiol (even at a cholestatic dose for hepatocytes) does not affect the secretory activities of rat intrahepatic biliary epithelium. ${ }^{30}$

In conclusion, our findings give further support to the role of endogenous estrogens as modulators of cholangiocyte proliferation in the course of cholestasis. In our opinion, estrogens act by synergizing the effects of growth factors in sustaining the proliferative machinery and depressing apoptosis. The fact that neither OVX nor exogenous estrogens has any effect on cholangiocyte proliferation in normal rats is consistent with this hypothesis because cholangiocytes are quiescent cells in the normal status ${ }^{6}$ and become proliferating only under certain experimental or clinical conditions where a complex network of growth factors is activated. ${ }^{7,9}$ In addition, when the estrogenic function was almost completely blocked by combining OVX and antiestrogens, cholangiocyte proliferation (evaluated by PCNA) still persists, and this indicates that, even in the absence of the estrogen synergizing effect, the growth factors still continue to exhibit their action, although at a lower level.

Cholangiocyte proliferation plays an important role as a repair and compensatory mechanism for bile duct loss during cholangiopathies, therefore, counteracting the evolution of the disease toward the terminal ductopenic stage. Estrogens and their metabolites have often been considered to have a pathogenic role in these diseases, which preferentially affects women. ${ }^{13,30}$ This is especially true for primary biliary cirrhosis, where an estrogenic functional deficiency has been hypothesized. ${ }^{13,31}$ Results from our study further highlight this hypothesis, as a mechanism favoring the clinical appearance or a more rapid progression of the disease, and encourage clinical studies aimed to find definitive demonstrations.

\section{References}

1. LaRusso NF. Pathobiology of biliary epithelia. In: Manns MO, Boyer JL, Jansen PLM, Reichen J, eds. Cholestatic liver diseases. London: Kluwer Academic Pub, 1998:62-67.

2. Boyer JL. Vanishing bile duct syndrome - from bench to bedside. In: Alvaro D, Benedetti A, Strazzabosco M, eds. Vanishing bile duct syndrome. London: Kluwer Academic Pub, 1997:240-246.

3. Desmet V, Roskams T, Van Eyken P. Ductular reaction in the liver. Pathol Res Pract 195;191:513-524.

4. Desmet V, Roskams T, Van Eyken P. Histopathology of vanishing bile duct diseases. Adv Clin Pathol 1998;2:87-99.

5. Desmet VJ, Roskams T, Van Eyken P. Pathology of the biliary tree in cholestasis: ductular reaction. In: Manns MO, Boyer JL, Jansen PLM, Reichen J, eds. Cholestatic liver diseases. Falk Symposium 102. London: Kluwer Academic Pub, 1998:143-154.

6. Alvaro D, Gigliozzi A, Attili AF. Regulation and deregulation of cholangiocyte proliferation. J Hepatol 2000;33:333-340. 
7. LeSage G, Alvaro D, Benedetti A, Glaser S, Marucci L, Baiocchi L, Eisel W, Caligiuri A, Phinizy JL, Rodgers R, Francis H, Alpini G. Cholinergic system modulates growth, apoptosis, and secretion of cholangiocytes from bile duct ligated rats. Gastroenterology 1999;117:191-199.

8. Alvaro D, Alpini G, Onori P, Perego L, Svegliati Baroni G, Franchitto A, Baiocchi L, Glaser SS, Le Sage G, Folli F, Gaudio E. Estrogens stimulate proliferation of intrahepatic biliary epithelium in rats. Gastroenterology 2000;119:1681-1691.

9. Glaser S, Benedetti A, Marucci L, Alvaro D, Baiocchi L, Caligiuri A, Chowdhury U, Papa E, Eisel W, Francis H, Phinizy JL, Alpini G. Gastrin inhibits cholangiocyte growth in bile duct ligated rats by interaction with CCK-B/gastrin receptors via $\mathrm{IP}_{3^{-}} \mathrm{Ca}^{2+}$, and PLC$\alpha$-dependent mechanisms. Hepatology 2000;32:17-25.

10. Eagon PK, Porter LE, Francavilla A, DiLeo A, Van Thiel DH. Estrogen and androgen receptors in liver: their role in liver disease and regeneration. Semin Liver Dis 1985;5:59-69.

11. Fisher B, Gunduz N, Saffer EAS, Zheng S. Relation of estrogen and its receptor to rat liver growth and regeneration. Cancer Res 1984;44:2410-2415.

12. Blum A, Cannon RO. Effects of oestrogens and selective oestrogen receptor modulators on serum lipoproteins and vascular function. Curr Opin Lipidol 1998;9:575-586.

13. Floreali A, Titta M, Plebani M, Faggian D, Chiaramonte M, Naccarato R. Sex hormones changes in post-menopausal women with primary biliary cirrhosis (PBC) and with cryptogenic chronic liver diseases. Clin Exp Obstet Gynecol 1991;18:229-234.

14. Stellon AJ, Williams R. Increased incidence of menstrual abnormalities and hysterectomy preceding primary biliary cirrhosis. $\mathrm{Br}$ Med J 1986;293:297-298.

15. Wolke AM, Schaffner F, Kapelman B, Sacks HS. Malignancy in primary biliary cirrhosis. High incidence of breast cancer in affected women. Am J Med 1984;76:1075-1078.

16. Goudie BM, Burt AD, Boyle P, MacFarlane G, Birnie GG, Mills PR, Gillis CR, MacSween RN, Watkinson G. Breast cancer in women with primary biliary cirrhosis. Br Med J 1985;291:1597-1598.

17. Hodgson SF, Dikson ER, Wahner HW, Johnson KA, Man KG, Riggs $\mathrm{BL}$. Bone loss and reduced osteoblastic function in primary biliary cirrhosis. Ann Intern Med 1985;103:855-860.

18. Olsson R, Mattsson LA, Obrant K, Mellstrom D. Estrogen-progestogen therapy for low bone mineral density in primary biliary cirrhosis. Liver 1999;19:188-192.

19. Crippin JS, Jorgensen RA, Dickson ER, Lindor KD. Hepatic osteodystrophy in primary biliary cirrhosis: effects of medical treatment. Am J Gastroenterol 1994;89:47-50.

20. Chen J, Robertson G, Field J, Liddle C, Farrell GC. Effect of bile duct ligation on hepatic expression of female-specific CYP2C12 in male and female rats. Hepatology 1998;28:624-630.

21. Carpino F, Gaudio E, Marinozzi G, Melis M, Motta PM. A scanning and transmission electron microscopic study of experimental extrahepatic cholestasis in the rat. J Submicrosc Cytol 1981;13: 581-598.

22. Gaudio E, Onori P, Pannarale L, Alvaro D. Hepatic microcirculation and peribiliary plexus in experimental biliary cirrhosis: a structural and ultrastructural study in the rat. Gastroenterology 1996;111:1118-1124.

23. Teutsch HF. Improved method for the histochemical demonstration of glucose-6-phosphatase activity. A methodological study. Histochemistry 1978;57:107-117.

24. Diel P, Smolnikar K, Michna H. The pure antiestrogen ICI 182780 is more effective in the induction of apoptosis and down regulation of BCL-2 than tamoxifen in MCF-7 cells. Breast Cancer Res Treat 1999;58:87-97.

25. Gandhi A, Holland PA, Knox WF, Potten CS, Bundred NJ. Effects of a pure antiestrogen on apoptosis and proliferation within human breast ductal carcinoma in situ. Cancer Res 2000;60: 4284-4288.

26. Newton CJ, Eycott K, Green V, Atkin SL. Response of estrogen receptor containing tumor cells to pure antiestrogens and the calmodulin inhibitor, calmidazolium chloride. J Steroid Biochem Mol Biol 2000;73:29-38.

27. Lau KM, LaSpina M, Long J, Ho SM. Expression of estrogen receptor (ER)- $\alpha$ and ER- $\beta$ in normal and malignant prostatic epithelial cells: regulation by methylation and involvement in growth regulation. Cancer Res 2000;60:3175-3182.

28. Weihua Z, Saji S, Makinen S, Cheng G, Jensen EV, Warner M, Gustafsson JA. Estrogen receptor (ER)- $\beta$, a modulator of ER- $\alpha$ in the uterus. Proc Natl Acad Sci U S A 2000;97:5936-5941.

29. Kullak-Ublick GA, Meier PJ. Mechanisms of cholestasis. Clin Liver Dis 2000;4:357-385.

30. Alvaro D, Gigliozzi A, Piat C, Carli L, Fraioli F, Romeo R, Francia C, Attili AF, Capocaccia L. Inhibition of biliary bicarbonate secretion in ethinyl estradiol-induced cholestasis is not associated with impaired activity of the $\mathrm{Cl}^{-} / \mathrm{HCO}_{3}{ }^{-}$exchanger in the rat. J Hepatol 1997;26:146-157.

31. Joplin R, Neuberger JM. Antigen expression in bile duct epithelia in cholestatic liver disease. In: Manns MO, Boyer JL, Jansen PLM, Reichen J, eds. Cholestatic liver diseases. Falk Symposium 102. London: Kluwer Academic Pub, 1998:226-238.

Received December 3, 2001. Accepted March 14, 2002.

Address requests for reprints to: Eugenio Gaudio, M.D., State University "La Sapienza," Department of Anatomy, via A. Borelli, 5000161 Rome, Italy. e-mail: eugenio.gaudio@uniroma1.it; fax: (39) 0649918062.

Supported by a grant from MIUR (Biomedicina, Cluster 04, progetto n.5, ex $60 \%$ and Cofin 2000 funds; to E.G.), a grant award from Scott \& White Hospital and Texas A\&M University (to G.A. and G.L.S.), NIH grant DK 54208 (to G.L.S.), NIH grant DK 58411 and VA Merit Award (to G.A.), a partial grant award from Scott \& White Hospital (to S.G.), and a MURST grant MM06215421/2 (40\% funds; to D.A. and A.F.A.). 